\title{
Determinação do uso do solo do município de Bandeirantes, Estado do \\ Paraná, através de imagem do Landsat 7 Etm+ \\ e técnicas de geoprocessamento ${ }^{1}$
}

\author{
Determination of the land use of the municipality of Bandeirantes, \\ Paraná State, using Landsat 7 Etm + image and \\ geoprocessing techniques
}

Teresinha Esteves da Silveira Reis ${ }^{2 *}$; Omar Neto Fernandes Barros ${ }^{3}$; Luiz Carlos Reis ${ }^{4}$

Resumo

\begin{abstract}
Para o planejamento agrícola de uma região, um dos itens fundamentais é a identificação das culturas instaladas. Os sistemas de informações geográficas, aliados a dados de sensores remotos são importantes ferramentas na identificação e avaliação do uso do solo. Com o objetivo de identificar o uso do solo do município de Bandeirantes - PR, utilizou-se as bandas 2, 3, 4 e 5 do LANDSAT 7 ETM+, NDVI (razão entre bandas 4 e 3). Para classificação da imagem, utilizou-se o sistema de classificação supervisionado por regiões, classificador Bhatta charya, implementado no SPRING 3.6.03 (Sistema de Processamento de Informações Georreferenciadas) desenvolvido pelo INPE (Instituto Nacional de Pesquisas Espaciais) e a composição colorida NDVI, 5 e 2 (R,G,B) contraste (média: $R=77,72, G=75,6, B=50,24$ ). $O$ uso do solo deste município refere-se principalmente a culturas anuais, pastagens e cana-de-açúcar, com participação de $27,31 \%, 20 \%$ e $18,31 \%$ respectivamente. A composição de bandas utilizada foi eficiente na determinação do uso do solo e a divisão entre bandas 4 e 3 para a obtenção do NDVI, foi importante para a detecção deste parâmetro, principalmente nas áreas de relevo mais acentuado. O sistema de informações geográficas demonstrou agilidade e precisão na avaliação das áreas temáticas.
\end{abstract}

Palavras-chave: Uso agrícola, sensoriamento remoto, sistema de informação geográfica (SIG)

\begin{abstract}
For the agricultural planning of a region, one of the fundamental items is the identification of the installed cultures. The geographic information systems, allied to remote sensing are important tools to the identification and evaluation of the land use. With the objective of identifying the land use of the municipal district of Bandeirantes-PR, it was used the bands 2, 3, 4 and 5 of LANDSAT 7 ETM+, NDVI (reason among bands 4 and 3 ). The image classification, was accomplished by the classification system supervised for areas, classifier Batha charya, implemented in SPRING 3.6.03, developed by INPE (National Spatial Research Institute) and the colored composition NDVI, 5 and $2(\mathrm{R}, \mathrm{G}, \mathrm{B})$ it contrasts (average: $\mathrm{R}=$ $77,72, \mathrm{G}=75,6, \mathrm{~B}=50,24)$. The municipal district land use ponders mainly in annual cultures, pasture and sugar cane, with participation of $27,31 \%, 20 \%$ and $18,31 \%$ respectively. The bands composition used was efficient in the determination of the land use, and the division among bands 4 and 3 to obtain NDVI was important, mainly in the areas of more irregular landscape. The geographical information system demonstrated agility and precision in the evaluation of the thematic areas.
\end{abstract}

Key words: Agricultural use, remote sensing, geographic information system (Gis)

${ }^{1}$ Parte da tese de doutorado da $1^{\mathrm{a}}$ autora.

${ }^{2}$ Prof. a Dra . da Faculdades “Luiz Meneghel”, UNESPAR. Bandeirantes, PR. e-mail reis@ffalm.br

${ }^{3}$ Prof. Dr. da Universidade Estadual de Londrina, PR. e-mail onbarros@uel.br

${ }^{4}$ Prof. da Faculdades “Luiz Meneghel”, UNESPAR. Bandeirantes, PR. e-mail reis@ffalm.br

*Autor para correspondência

Recebido para publicação 06/04/04 Aprovado em 15/10/04 


\section{Introdução}

Informações confiáveis sobre culturas instaladas, área plantada ou sua distribuição espacial dentro de uma determinada região, são fundamentais para direcionar o planejamento e definição de prioridades por parte do poder público e privado envolvidos na atividade agrícola.

Os sistemas de informações geográficas são importantes ferramentas para a identificação e avaliação de áreas ocupadas por propriedades de diferentes portes e pela vegetação natural, além do monitoramento anual do desflorestamento em certas regiões (ASSAD, 1998; SILVA NETO, 1996; SHIMABUKURO et al., 1998).

Andrade et al. (1998), salienta que a análise visual de imagens consiste basicamente na inspeção e identificação de diferentes padrões tonais /cor e texturais em cada banda, e na sua comparação em diferentes bandas e épocas. Assim, dois aspectos devem ser levados em consideração: o aspecto espectral e o temporal das imagens. $\mathrm{O}$ espectral permite a avaliação de informações em diversas bandas. Desta forma, as características espectrais dos alvos podem ser registradas de modos diferentes nas diversas bandas espectrais existentes, possibilitando a identificação de diferentes alvos através de comparação entre bandas.

Segundo Valério Filho e Ferreira Pinto (1996), os alvos da superfície terrestre podem ter sua resposta espectral alterada com o tempo, em função de fatores externos ou próprios de sua natureza. Dentre estes alvos, a cobertura vegetal é tida como a mais sujeita a modificações intrínsecas. Os mesmos autores salientam a importância da interação energia solar com a planta na aplicação de dados de sensoriamento remoto para estudo da dinâmica da ocupação do solo.

O sistema LANDSAT foi o primeiro programa operacional de sensoriamento remoto orbital, sendo que o LANDSAT 7 foi lançado em 1999, com sensor denominado ETM+ (Enhanced Thematic Mapper Plus). As informações do LANDSAT 7 ETM+ podem ser obtidas por 8 bandas espectrais, sendo as bandas 1,2,3,4,5 e 7 com resolução espacial de $30 \mathrm{~m}$, a banda 6 com 60 m e a 8 com $15 \mathrm{~m}$. Desta forma é possível a utilização destas imagens em estudos na escala de até 1:25.000.

Os temas de interesse para o estudo do uso do solo geralmente concentram-se nas determinações das diferentes densidades e coberturas vegetais, dos tipos de solos e respectivos manejos, do uso urbano e da água.

As técnicas de processamento de imagens digitais podem ser classificadas em três conjuntos (NOVO, 1998; INPE, 1999): técnicas de pré-processamento, técnicas de realce e técnicas de classificação.

As técnicas de pré-processamento referem-se ao conjunto de programas que permitem a transformação de dados digitais brutos em dados corrigidos geometricamente.

As técnicas de realce melhoram a qualidade visual da imagem com conseqüente melhora na interpretação visual da mesma. Dentre estas, para a realização do objetivo deste trabalho, destacam-se a manipulação de contraste e operações entre bandas. Nesta última, utiliza-se uma ou duas bandas de uma mesma área geográfica, previamente georreferenciadas. Realiza-se a operação "pixel" a "pixel", através de uma regra matemática definida, tendo como resultado uma banda representando a combinação das bandas originais. Estas operações permitem a compressão de dados, diminuindo o número de bandas, mas pode ocasionar perda da informação original. Estas operações podem requerer um fator de ganho (multiplicativo) ou "off-set" (aditivo), para melhorar a qualidade de contraste da imagem. De maneira geral, a operação de adição realça a similaridade entre bandas ou diferentes imagens e, a subtração, a multiplicação e divisão, realçam diferenças espectrais entre as bandas. A operação de divisão de imagens consiste numa operação não-linear. É utilizada para realçar as diferenças espectrais de um par de bandas, caracterizando determinadas feições da curva de assinatura espectral de alguns alvos. Para aumentar 
o contraste entre solo e vegetação, pode-se utilizar a razão entre bandas referentes ao vermelho e infravermelho próximo, constituindo assim, os chamados índices de vegetação normalizada (NDVI), conforme manual do SPRING (INPE, 1999). Jorge e Garcia (1997) utilizaram esta técnica de realce no estudo da avaliação da fragmentação florestal e da distribuição dos espaços verdes na área urbana, obtendo resultados satisfatórios.

Classificação é o processo de extração de informação em imagens para reconhecer padrões e objetos homogêneos. A informação espectral de uma cena pode ser representada por uma imagem espectral, onde cada "pixel" tem as coordenadas espaciais $\mathrm{x}$, y e a coordenada espectral $\mathrm{z}$, que representa a radiância de um alvo no intervalo de comprimento de onda de uma banda espectral. Cada "pixel" de uma banda possui uma correspondência espacial com um outro "pixel", em todas as outras bandas, ou seja para uma imagem de $\mathrm{n}$ bandas, existem n níveis de cinza associados à cada "pixel", sendo um para cada banda espectral. As técnicas de classificação visam o reconhecimento automático de objetos da cena a partir da análise quantitativa dos níveis de cinza (NOVO, 1998).

Os métodos de classificação podem ser divididos em métodos supervisionados e não supervisionados. No primeiro, é necessário a interação com o sistema para se determinar um conjunto de amostras referentes aos temas de interesse, e no segundo o sistema determina, a partir de algoritmos próprios, o conjunto de amostras para identificar os mais variados temas. Nos supervisionados, pode-se ter classificadores por pixel, onde se utiliza apenas a informação espectral, isoladamente, de cada pixel para achar regiões homogêneas ou classificadores por regiões que utilizam, além de informação espectral de cada "pixel", a informação espacial que envolve a relação entre os "pixels" e seus vizinhos, procurando simular o comportamento do fotointérprete, ao reconhecer áreas homogêneas de imagens baseados nas propriedades espectrais e espaciais da imagem. A informação de borda é utilizada inicialmente para separar as regiões e as propriedades espaciais e espectrais que irão unir áreas com mesma textura (INPE, 1999).

Este trabalho teve por objetivo determinar o uso do solo do município de Bandeirantes por meio do uso das bandas do LANDSAT 7 ETM+ e os métodos de processamento de imagem do SPRING 3.6.03 (Sistema de Processamento de Informações Georreferenciadas) desenvolvido pelo INPE (Instituto Nacional de Pesquisas Espaciais).

\section{Material e Métodos}

Utilizou-se a imagem do satélite LANDSAT 7 ETM+, WRS 221_76 de 15/04/2001, SAD 69 projeções UTM. A área de estudo localiza-se nas coordenadas $50^{\circ} 29^{\prime} 44,08^{\prime \prime}-50^{\circ} 09^{\prime} 42,56^{\prime \prime} \mathrm{W}$ e $23^{\circ}$ $17^{\prime} 5.10^{\prime \prime}-23^{\circ} 00^{\prime} 58.40^{\prime}$ ' S, cujo retângulo envolvente compreende área de $1024,71 \mathrm{Km}^{2}$. Trabalhou-se com as bandas originais 2, 3, 4 e 5 e com NDVI (razão entre as bandas 4 e 3). Em seguida procedeu-se à segmentação de imagem, onde a mesma é dividida em regiões que correspondem às áreas de interesse de aplicação. Entende-se por regiões um conjunto de "pixels" contíguos que se espalham bidirecionalmente e que apresentam uniformidade. As regiões foram obtidas através do método crescimento de regiões, por promover o agrupamento de dados, em que somente as regiões adjacentes espacialmente podem ser agrupadas. Inicialmente, este processo de segmentação rotula cada "pixel" como uma região distinta. Calcula-se um critério de similaridade para cada par de regiões adjacentes espacialmente. $\mathrm{O}$ critério de similaridade baseia-se em um teste de hipótese estatístico que testa a média entre as regiões. A seguir, divide-se a imagem em um conjunto de sub-imagens e então se realiza a união entre elas, segundo um limiar de agregação definido. Utilizaram-se as amostras de treinamento para estimar a função densidade de probabilidade para as classes apontadas e requer interação do usuário, através do treinamento. Neste caso, as amostras serão as regiões formadas na segmentação de 
imagens que foi realizada pelo processo de crescimento de regiões com limiar de similaridade igual a 8 e de agregação igual a 10. Adotou-se o limiar de aceitação das amostras de 95\%. Posteriormente, a classificação de imagem foi realizada através do método supervisionado por meio do classificador Bhatta charya.

Considerou-se para a classificação a composição colorida NDVI, 5 e 2 (R,G,B) contraste (média: $\mathrm{R}=$ $77,72 ; \mathrm{G}=75,6 ; \mathrm{B}=50,24)$.

Dentre as técnicas de realce disponíveis no programa SPRING 3.6.03, realizou-se, além do realce imagem, a divisão entre as bandas $3(0,63-0,69$ $\mathrm{mm})$ e $4(0,76-0,90 \mathrm{~mm})$ com o objetivo principal de realçar as diferenças espectrais entre as bandas, realçar solo e vegetação, diminuir o efeito de sombreamento causado pelo fator topográfico e determinar o índice de vegetação normalizada (NDVI), conforme equação:

$\mathrm{C}=\mathrm{G} \times((\mathrm{A}-\mathrm{B}) /(\mathrm{A}+\mathrm{B}))+\mathrm{O}$, sendo:

$\mathrm{C}=\mathrm{NDVI}$ (índice de vegetação normalizada)

$\mathrm{G}=$ fator ganho

$\mathrm{O}=$ fator offset

$\mathrm{A}=$ banda infravermelho próximo (banda 4)

$\mathrm{B}=$ banda vermelho ( banda 3 )

As amostras de cada tema foram previamente determinadas e foram obtidas através de suas coordenadas e do dispositivo do SPRING em posicionar cursor.

Os temas considerados mais relevantes para a avaliação do uso do solo da região foram:

a) mata: a análise da imagem permitiu a identificação de vários fragmentos florestais.

b) cana-de-açúcar: a presença de uma importante agroindústria do setor de açúcar e álcool no município tornou esta cultura de grande importância na economia da região;

c) pastagem: na análise deste tema considerou-se pasto limpo como sendo áreas onde a cobertura vegetal era constituída apenas de gramíneas para o consumo do gado, não havendo outra forma de vegetação, e pasto sujo como sendo aquelas onde se constatou a presença de outros tipos de vegetação, caracterizando certo abandono da área para uso como pastagem, inclusive manchas de capoeira;

d) culturas anuais: considerando a época da obtenção da imagem, neste tema observou-se que as culturas de verão predominantes na região (soja e milho), estavam com seu ciclo concluído, ou seja, estavam por colher ou já haviam sido colhidas. Neste caso considerou-se para efeito de análise três níveis quanto ao seu ciclo: cultura anual 1 , com alto volume de palha, quer seja lavoura colhida ou por colher; cultura anual 2 , com médio volume de palha; e cultura anual 3 , com pouco ou nenhuma palha;

e) café: a região foi grande produtora de café até a década de 1970. Por questões climáticas e econômicas, tal atividade sofreu forte declínio, porém atualmente observa-se incentivo para o retorno desta atividade.

f) solo exposto: neste tema foram consideradas as áreas conduzidas pelo sistema convencional, que em sua maioria seriam destinadas para as culturas de inverno;

g) uso urbano: este tema não foi analisado pelos dados orbitais, pois se considerou que, sendo o objetivo preliminar deste estudo a verificação da aptidão e a compatibilidade de uso do solo agrícola, tal tema deveria ser descartado na análise orbital. Como o uso urbano do solo é muito diversificado, a inclusão dos "pixels" deste tema poderiam dificultar a classificação da imagem. Por este motivo, optouse por implantação de uma máscara sobre a área referente ao perímetro urbano, extraída do programa Paraná Cidade.

h) Outros usos: neste tema estão incluídos todos os usos não descritos acima. Constitui-se principalmente de fruticultura, áreas em pousio, piscicultura e culturas diversas, tais como alfafa, mandioca e etc.

\section{Resultados e Discussão}

Na Figura 1, referente à imagem LANDSAT 7 ETM+, observa-se o aspecto geral da região contida no retângulo envolvente e os limites do perímetro urbano, sobre o qual efetuou-se a máscara. 
Figura 1: Imagem Landsat 7 ETM+, composição colorida NDVI(R), 5(G), 2(B), do município de Bandeirantes-Pr.

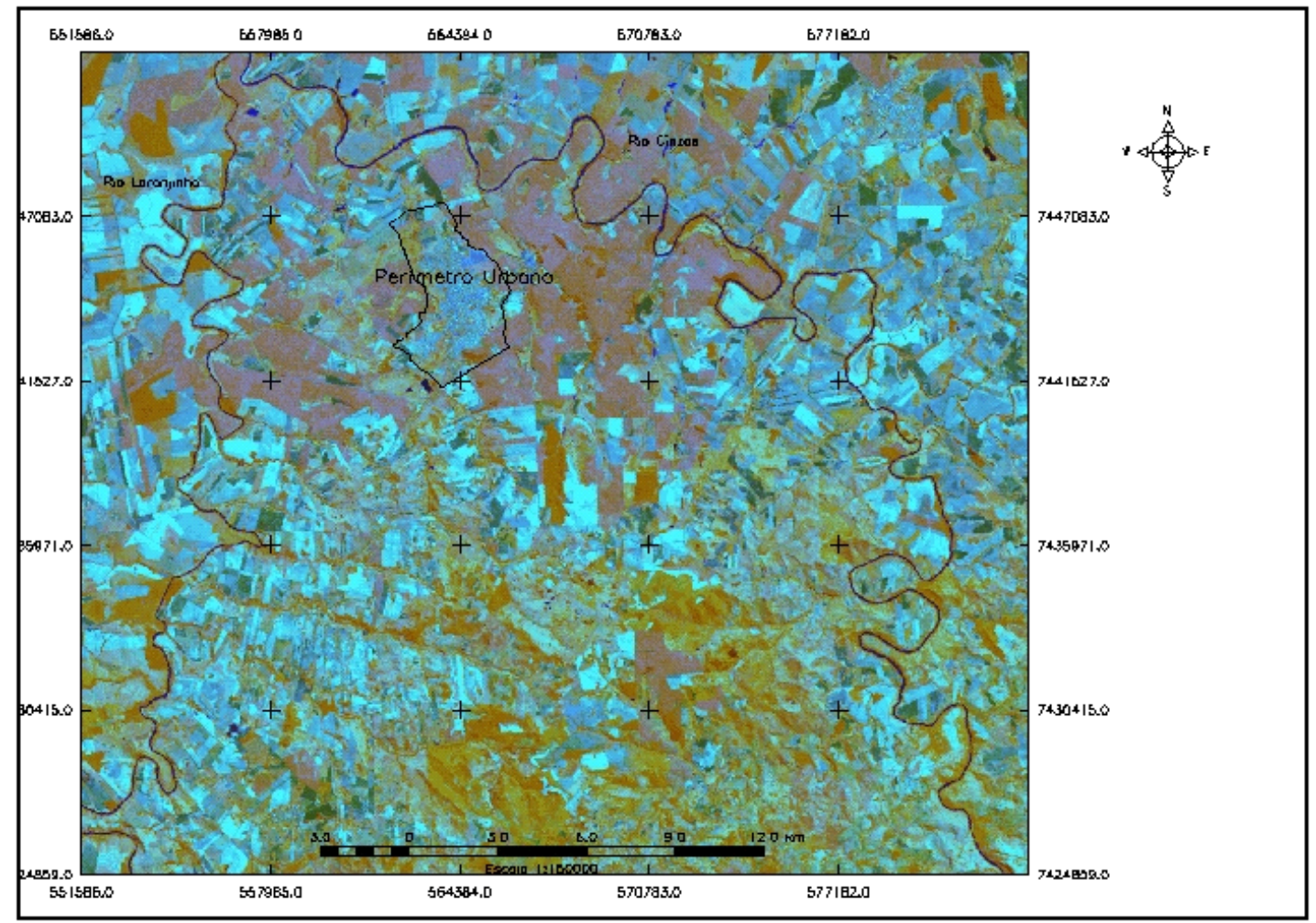

Vários autores trabalharam com as bandas 3, 4 e 5 com o intuito de identificar o uso do solo, como Silva et al.(1993), Shimabukuro et al. (1998), Sano et al. (1998) e Rodrigues et al. (2001). Tais bandas abrangem os comprimentos de ondas referentes aos principais elementos de análise para o uso do solo. No entanto, ao determinar o índice de vegetação normalizada (NDVI), obtido através da divisão entre as bandas 3 e 4, pode-se incorporar a banda 2 no conjunto de bandas para análise. A vantagem deste procedimento está na possibilidade de melhor identificar as diferentes coberturas vegetais, quer seja pela biomassa ou estágios vegetativos das culturas. Pelas condições de relevo observadas na área de estudo, conforme Reis (2003), é de se supor que o NDVI apresente maior possibilidade de detecção dos alvos, uma vez que, realça as diferenças espectrais entre as bandas $3(0,63-0,69 \mathrm{~mm})$ e $4(0,76-0,90$ $\mathrm{mm}$ ), diminui o efeito de sombreamento causado pelo fator topográfico e realça solo e vegetação, esta última importante por haver culturas em vários estágios e diferentes sistemas de implantação. Jorge e Garcia (1997), utilizaram esta técnica no mapeamento de uso do solo e constataram ser importante para a diferenciação da biomassa.

Previamente à classificação é necessária uma análise da qualidade das amostras utilizadas na segmentação. Nesta análise verificou-se que o tema de maior confusão foi o uso urbano, fato atribuído à diversidade de alvos, tendo se observado que parte das amostras foram enquadradas em solo exposto, cultura anual e pastagem. Coutinho et al. (1998) e Rodrigues et al. (2001) atribuíram a dificuldade na classificação do uso urbano à variabilidade dos tipos de cobertura observadas nestas áreas. Por este fato optou-se pela máscara sobre o perímetro urbano, uma vez que o objeto principal deste estudo ser o uso agrícola.

No modelo fundiário do município observou-se que as maiores propriedades caracterizam-se por uma maior uniformidade no uso do solo, ou seja, predominantemente com cana e culturas anuais como 
soja, seguida posteriormente por milho. Nas pequenas propriedades a diversidade no uso do solo é maior, ou seja, pequenas áreas de pastagem, café e culturas de subsistência. Isto pode acarretar maior dificuldade ao sistema para a detecção de pequenas áreas relativas a estes temas, uma vez que a resolução espectral do sensor é de $30 \mathrm{~m}$ e também pelo fato de alguns temas se encontrarem dispersos na paisagem. Pereira et al. (1998), constataram que os temas dispersos na paisagem apresentaram o pior desempenho na classificação da imagem orbital. Venturini e Santos (1998) advertem que a presença de pixels isolados dentro das classes ocasiona perda de uniformidade da imagem, que pode dificultar a interpretação e avaliação temática e gerar imagens temáticas fragmentadas.

A Tabela 1 e Figura 2 demonstram o resultado da classificação realizada para a área de estudo. O uso de solo apresenta predominância das culturas anuais, pastagem e cana-de-açúcar, com 27,31\%, 20,00\% e $18,31 \%$ da área do município, respectivamente. Convém ressaltar, que grande parte das áreas avaliadas como solo exposto e outros usos incluem áreas destinadas para culturas anuais.

Tabela 1: Dados demonstrativo do uso do solo no município de Bandeirantes-PR.

\begin{tabular}{|c|c|c|c|}
\hline & \multicolumn{3}{|c|}{ Área } \\
\hline & $\mathrm{Km}^{2}$ & ha & $\%$ \\
\hline \multicolumn{4}{|l|}{ Uso do Solo } \\
\hline Cult. Anuais (níveis 1,2 e 3 ) & 120,28625 & $12.028,625$ & 27,31 \\
\hline Pastagem (pasto sujo + pasto) & 88,12687 & $8.712,687$ & 20,00 \\
\hline Cana -de-açúcar & 80,67750 & $8.067,75$ & 18,31 \\
\hline Café & 28,46437 & $2.846,437$ & 6,46 \\
\hline Solo exposto ${ }^{1}$ & 18,98312 & $1.898,312$ & 4,31 \\
\hline Mata & 21,65187 & $2.165,187$ & 4,92 \\
\hline Uso urbano & 15,21014 & $1.521,014$ & 3,45 \\
\hline Outros usos ${ }^{2}$ & 67,10294 & $6.710,294$ & 15,24 \\
\hline Total área município & 440,50309 & $44.050,309$ & 100 \\
\hline
\end{tabular}

${ }^{1}$ uma parcela expressiva deste tema deve ser destinada para culturas anuais, uma vez tratar-se de terras conduzidas por manejo convencional e a época da imagem refere-se ao período de implantação das culturas de inverno.

${ }^{2}$ neste tema os usos são muito variados, observando-se áreas em pousio, fruticultura, piscicultura e culturas diversas.
O monitoramento agrícola de uma região, baseado na quantificação e classificação da área plantada e tipo de cultura, através de dados extraídos de sensores remotos e manipulados em um sistema de informação geográfica, necessita de integração com amostras de campo (SANO et al, 1998). Na composição colorida NDVI-5-2 (R,G, B), observou-se várias tonalidades referentes ao tema culturas anuais. $\mathrm{Na}$ análise in loco, verificou-se tratar de estágios diferentes das culturas anuais predominantes na região, soja e milho. A imagem foi obtida em meados do mês de abril, onde estas culturas encontravam-se com seu ciclo vegetativo concluído. Os diferentes tons encontrados eram em função do volume de palha observados na superfície, ou seja, culturas por colher, aquelas já colhidas e áreas com remanescentes de palhada e áreas recém plantadas com culturas de inverno. Por esta razão, para a classificação deste tema foram considerados, no sistema, três tipos diferentes de conjunto de amostras.

As áreas ocupadas com cana-de-açúcar apresentaram padrões tonais e texturais mais homogêneos. A safra no município normalmente inicia-se a partir da segunda quinzena do mês de abril, portanto a cultura encontrava-se com certa uniformidade na biomassa na época da aquisição da imagem. Outro elemento observado foi o fato dessa lavoura ocupar áreas extensas, sobretudo na região norte do município. Venturini e Santos (1998) salientam que temas que ocupam contínuas e largas extensões nas imagens, evitando assim o efeito de borda, possibilitam aquisição de amostras com valores espectrais mais fidedignos da classe, facilitando a classificação dos mesmos.

Para o tema pastagem foi necessária a obtenção de dois conjuntos distintos de amostras, pasto limpo e pasto sujo. Observou-se no campo diversidade na qualidade da cobertura vegetal, quer seja pela espécie da gramínea utilizada e ou pelo manejo, uma vez que muitas áreas encontravam-se com certo grau de abandono, havendo inclusive a formação de capoeira. Áreas de pastagens e café encontravam-se normalmente em áreas de relevo mais acentuado na 
porção sul do município, onde há possivelmente, conforme relatado por Rodrigues et al. (2001), influência do fator topográfico na reflectância dos pixels destas áreas. No entanto, a operação de divisão entre bandas, realizada através das bandas $3(0,63-$ $0,69 \mathrm{~mm})$ e $4(0,76-0,90 \mathrm{~mm})$ diminuem este efeito, permitindo melhores resultados na classificação.

Figura 2: Mapa de uso do solo do município de Bandeirantes-PR.

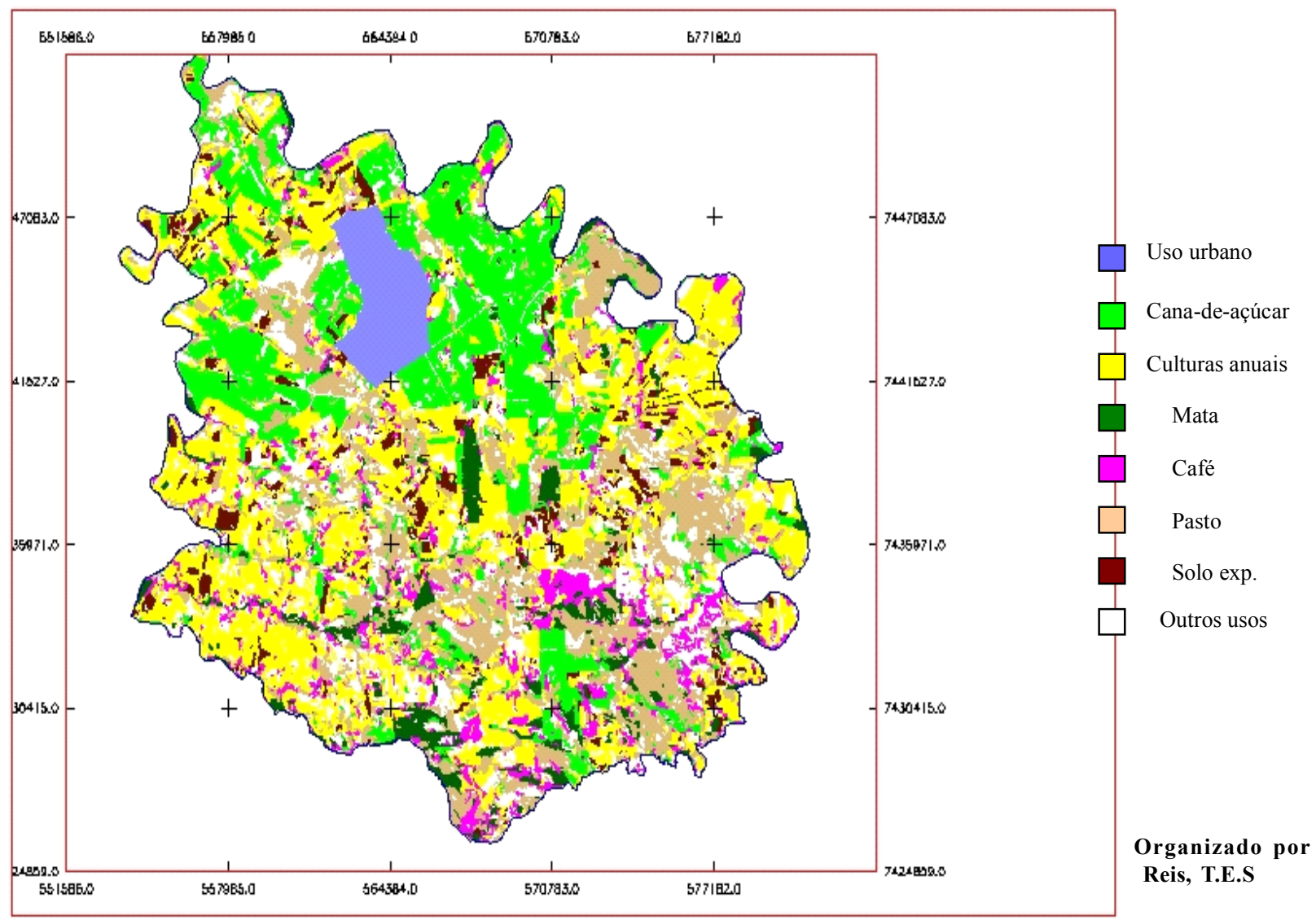

As áreas de mata representam um pouco mais que 2.000 ha, aproximadamente $5 \%$ da área do município. Observou-se completa ausência de fragmentos florestais nas regiões mais planas, de melhor aptidão para a atividade agrícola, e em longos trechos de córregos e rios. Segundo o Código Florestal Brasileiro, as matas ciliares deveriam ocupar uma faixa marginal de no mínimo $30 \mathrm{~m}$ de largura, o que sistematicamente não ocorre no município.

Solo exposto foi outro tema que apresentou homogeneidade nas amostras. Este tema pode ser considerado como áreas que serão destinadas às culturas de inverno, pelo manejo convencional. Porém, não se pode afirmar que as áreas restantes, com a mesma finalidade, sejam conduzidas por outro manejo, como por exemplo, o plantio direto.

Os usos variados do solo que não foram identificados através do conjunto amostral de cada tema, foram enquadrados no tema outros usos, estando localizados de forma dispersa em todo o município. In loco pôde-se observar que a maioria refere-se a áreas em pousio, fruticultura variada, como cultivo de banana e uva e pequenas áreas de alfafa e mandioca. 


\section{Conclusões}

O uso do solo do município concentra-se principalmente em culturas anuais, pastagem e canade-açúcar.

A composição de bandas utilizadas foi eficiente na determinação do uso do solo. A divisão entre bandas para a obtenção do NDVI foi importante para a detecção do uso do solo, principalmente nas áreas de relevo mais acentuado.

O sistema de informações geográficas demonstrou agilidade e precisão na avaliação das áreas temáticas.

\section{Referências}

ANDRADE, N. S. O.; ARAUJO, L. S.; NUMATA, I. Estudo da dinâmica da cobertura vegetal e uso da terra na região de Ji-Paraná. In: SIMPÓSIO BRASILEIRO DE SENSORIAMENTO REMOTO, 9., 1998, Santos. Anais... Santos, 1998.

ASSAD, M. L. L. Sistemas de informações geográficas na avaliação da aptidão agrícola de terras. In: ASSAD, E. D.; SANO, E. E., (Ed.). Sistema de Informações Geográficas: aplicações na agricultura. Planaltina: EMBRAPA-CPAC, 1998. p. 173-199.

ASSAD, M. L. L.; HAMADA, E.; CAVALIERI, A. Sistema de informações geográficas na avaliação de terras para a agricultura. In: ASSAD, E. D.; SANO, E. E. (Ed.) Sistema de Informações Geográficas: aplicações na agricultura. Planaltina: EMBRAPA-CPAC, 1998. p. 191-232.

COUTINHO, A. C.; MIRANDA, E. E.; MIRANDA, J. R. Mapeamento da superfície terrestre através da utilização do método de segmentação por crescimento de regiões e classificação supervisionada de imagem de satélite. In: SIMPÓSIO BRASILEIRO DE SENSORIAMENTO REMOTO, 9., 1998, Santos. Anais... Santos, 1998

INPE. Tutorial - Spring Versão Windows, São José dos Campos, 1999.

JORGE, L. A. B.; GARCIA, G. J. A study of habitat fragmentation in southeastern Brazil using remote sensing and geographic information systems. Forest Ecology and Management. Amsterdam, v. 98, n. 1, p. 35-47, 1997.

LOCH, R. E. N.; KIRCHINER, F. F. Classificação de imagens multiespectrais Landsat TM e feições de textura: mapeamento da cobertura vegetal. Floresta. Curitiba, v. 27, n. $1 / 2$, p. $41-58,2000$.
NOVO, E. M. L. M. Sensoriamento Remoto: princípios e aplicações. 2.ed. São Paulo: Edgard Blücher, 1998.

PEREIRA, N. R.; SCHUBACK, P. A.; CARVALHO JUNIOR, W. O uso de técnicas de geoprocessamento na detecção de áreas de incompatibilidade de uso, com base na aptidão agrícola das terras e o uso atual - estudo aplicado no município Eng ${ }^{\circ}$ Paulo Frotim. In: SIMPÓSIO BRASILEIRO DE SENSORIAMENTO REMOTO, 9., 1998, Santos. Anais... Santos, 1998.

REIS, T. E. S. Avaliação dos remanescentes florestais no período de 1970 a 1997 e dos parâmetros físico-químicos de Nitossolo Vermelho sob diferentes usos no municipio de Bandeirantes-PR. 2001. Dissertação (Mestrado)Universidade Estadual de Londrina, Londrina, 2001.

RODRIGUES, J. B. T.; ZIMBACK, C. R. L.; PIROLI, E. L. Utilização de sistemas de informação geográfica na avaliação do uso da terra em Botucatu (S.P). Revista Brasileira de Ciência do Solo, Campinas, v. 25, p. 675681,2001.

SANO, E. E.; ASSAD, E. D.; ORIOLI, A. L. Monitoramento da ocupação agrícola. In: ASSAD, E. D.; SANO, E. E., (ed.) Sistema De Informações: aplicações na agricultura. Planaltina: EMBRAPA-CPAC,1998. p. 179-190

SHIMABUKURO, Y. E.; BATISTA, G. T.; MELLO, E. M. K.; MOREIRA, J. C.; DUARTE, V. Using shade fraction image segmentation to evaluate deforestation in Landsat Thematic Mapper images of the Amazonian region. International Journal Remote Sensing, London, v. 19, n. 3, p. 535-541, 1998.

SILVA NETO, A. F.; BARBOSA, M. P.; SANTOS, M. J. Avaliação do desmatamento na bacia do alto Rio Sucuru, com base em imagens TM/LANDSAT-5. Engenharia Agrícola, Sorocaba, v. 16 n. 1, p. 1-7, 1996.

VALÉRIO FILHO, M.; PINTO, S. A. F. Imagens orbitais aplicadas ao levantamento de dados do meio físico: contribuição ao planejamento de microbacias hidrográficas. In: CONGRESSO BRASILEIRO E ENCONTRO NACIONAL DE PESQUISA SOBRE CONSERVAÇÃO DO SOLO, 8., 1996, Londrina Anais... Londrina: IAPAR, 1996, p. 77-95.

VENTURINI, A.; SANTOS, J. R. Técnicas de classificação de imagens para análise de cobertura vegetal. In: ASSAD, E. D.; SANO, E. E. (Ed.). Sistema de Informações: aplicações na agricultura. Planaltina: EMBRAPA-CPAC, 1998. p. 351-378.

RODRÍGUEZ, J. B. T; ZIMBACK, C. R. L.; PIROLI, E. L. Utilização de sistema de informação geográfica na avaliação do uso da terra de Botucatu (SP). RevistaBrasileira de Ciencia.do Solo, Campinas, v. 25, p. 675-681, 2001. 